\title{
PSYCHODIDAE (DIPTERA) DEL PERÚ I: PHLEBOTOMINAE EN HUÁNUCO, PASCO Y CUSCO, SU RELACIÓN CON LA ENFERMEDAD DE CARRIÓN Y LA LEISHMANIOSIS TEGUMENTARIA
}

\section{PSYCHODIDAE (DIPTERA) OF PERU I: PHLEBOTOIMINAE OF HUANUCO, PASCO AND CUSCO, ITS RELATION TO CARRION'S DISEASE AND DERMAL LEISHMANIOSIS}

\author{
Abraham G. Cáceres(1/*), Eunice A. B. Galati(2), José Pinto(3), Roberto Paredes(4), Robert \\ Reátegui(3), Jorge Pérez(3), Luzmila Chevarría(5), Henry Yáñez(5) y Víctor Zorrilla(6).
}

\section{RESUMEN}

A las 120 especies de flebotomineos reportadas hasta 1999 para el Perú, se adiciona: Brumptomyia quimpen, Evandromyia sallesi, Lutzomyia scorsai, L. rispaili, L. wattsi, Micropygomyia longipennis, Trichophoromyia howardi, $T$. arevaloi, Trichopygomyia turelli, $W$. euniceae y $W$. leponti, incrementando a 131 especies el número de flebotomíneos.

Se da a conocer la distribución geográfica de flebotomíneos en los departamentos de Huánuco, Pasco y Cusco; además, se destaca nuevos reportes de flebotomíneos en los departamentos de Ayacucho, Amazonas, Cajamarca, Huancavelica, Junín, La Libertad, Loreto y San Martín. Así mismo, se presentan evidencias epidemiológicas de algunos flebotomíneos de los subgéneros Helcocyrtomyia y Pifanomyia como probables vectores de agentes patógenos de la leishmaniosis tegumentaria y de la enfermedad de Carrión (verruga peruana).

Palabras clave: Phlebotominae, Perú, Leishmaniosis cutánea andina (uta), enfermedad de Carrión

\section{ABSTRACT}

One hundred and twenty species had been reported up to 1999 and the following 11 are now added to Peruvian phlebotomine fauna: Brumptomyia quimperi, Evandromyia sallesi, Lutzomyia rispaili, $L$. scorzai, L. watsi, Micropygomyia longipennis, Trichophoromyia howardi, T. arevaloi, Trichopygomyia turelli. Warileya euniceae and W. leponti. A complete list of species in Huanuco, Pasco and Cusco departments is included and new records for Ayacucho, Amazonas, Cajamarca, Huancavelica, Junin, La Libertad, Loreto and San Martin are given.

There appears to be epidemiological evidences of some phlebotomines of the subgenera Helcocyrtomyia and Pifanomyia as likely vectors of dermal leishmaniosis and Carrion's disease.

Key words: Phlebotominae, Peru, dermal leishmaniosis and Carrion's disease.

\section{INTRODUCCIÓN}

Los flebotomíneos son dípteros de importancia mundial en salud pública, por ser vectores de agentes patógenos de la leishmaniosis tegumentaria y visceral (Young

(1) Departamenlo de Mic robiologia Medica, Facultad de Medicina e Inst!luto de Medicina Tropical «Daniel A. Carrionn. Universidad Nacional Mayor de San Marcos: (2) Deparlamento de Epidemıologia, Faculdade de Saude Pública, Universidade de Saco faulo, Brasil, (3) Unidad herrilorial de Salud de Tingo Maria, Huáriuco, (4) Hespial de La Merced, Chanchamayo. Junin; (5) Laboratorio leterencial cie la Reg on de Salud Cusco: (6) Facultad de Ciencias Biclo. gicas. Universidad Nacional Mayor de San Marcos.

- Correspondencia: Abraham Cáceras: Sección de Entomologia del Inslituto de Medicina Tropicál "Daniel A. Carricrin. UNMSM

Telèt: +51-1-452.4124:+51-1-254.4027. Fax: $+51-1-2545656$

E-mail: acaceres 31 : 'ilma 4 com y Arias, 1992; Young \& Duncan, 1994), de la enfermedad de Carrión, conocida como verruga peruana (Townsend, 1913; Hertig, 1942; Cáceres, 1993) y de Arbovirus (Tesh, 1988).

Los flebotomíneos están presentes en todos los continentes y constituyen más de 800 especies descritas, las cuales fueron divididas en 1991 por Artemiev en tribus Idiophlebotomini y Phlebotomini; con representantes en el Viejo Mundo (VM), Nuevo Mundo (NM) y Oceanía (OC). En 1995, Galati también considera las dos tribus, sustituyendo 
el nombre de Idiophlebotomini por Hertigiini. En esta clasificación la tribu Phlebotomini está constituida por seis subtribus: Phlebotomina (VM), Australophlebotomina (OC), Brumptomyiina (NM), Sergentomyiina (VM y NM), Lutzomyiina (NM) y Psychodopygina (NM). Las especies de flebotomíneos que tienen importancia en salud pública pertenecen a la tribu Phlebotomini.

En América se han reportado aproximadamente 459 especies, y varios géneros y subgéneros de Lutzomyiina y Psychodopygina son considerados como vectores de zoonosis $y / o$ antropozoonosis.

Hasta 1999 se han reportado 120 especies de flebotomíneos para el Perú. Se supone que, a medida que se continúen explorando nuevas áreas, el número sería mayor; pues, pocas áreas de los valles interandinos y del llano amazónico han sido exploradas.

Existen varios nombres populares regionales para los flebotomíneos adultos, mencionado por Llanos (197I) a esta lista de nombres se adiciona los siguientes: «angelillos» (Huánuco), «capa blanca» (Ayacucho); "pumamanchachi», "pumakanikum»y «chuspi» (Áncash); «lalapo» (Cajamarca) y «quitis» (Cusco).

En el Perú, las especies de flebotomíneos señalados como vectores de leishmaniosis cutánea andina «uta» pertenecen a los subgéneros Helcocyrtomyia y Pifanomyia (Cáceres, 1995), respectivamente de los géneros Lutzomyia y Pintomyia que pertenecen a la tribu Lutzomyiina de acuerdo a Galati (1995). Algunas especies de Pifanomyia también están relacionadas como vectores de Ja enfermedad de Carrión (Townsend, 1913; Hertig, 1942; Cáceres, 1993; Cáceres et al., 1997).

El presente trabajo es el primero de una serie de contribuciones bajo el título «Psychodidae (Díptera) del Perú», donde se dará a conocer la distribución geográfica de los flebotomíneos por provincias y la relación existente con la enfermedad de Carrión (veruga peruana) y la leishmaniosis tegumentaria. En esta oportunidad se da a conocer la distribución geográfica de flebotomíneos para Huánuco, Pasco y Cusco, donde la leishmaniosis cutánea andina «uta»y mucocutánea es endémica; mientras que la enfermedad de Carrión (verruga peruana) está presente en Huánuco (Vizcarra et al., 1999) y en Cusco según Nuñez et al., 1999 y (Montoya et al., 1999); así mismo, se da nuevos reportes para los departamentos de Ayacucho, Amazonas, Cajamarca, La Libertad, San Martín, Junín, Huancavelica y Loreto.

\section{MATERIAL Y MÉTODOS}

La lista de flebotomíneos identificados, que se presenta para Huánuco, Pasco y Cusco, se basó en trabajos publicados o comunicados por investigadores nacionales y extranjeros; así como en ejemplares capturados por los autores en zonas verrucógenas y utógenas de los tres departamentos (Fig. 1).

Los estudios taxonómicos se basaron exclusivamente en la identificación morfológica, para lo cual los adultos fueron clarificados y montados en resina entre lámina y laminilla permanentes para microscopio, según la técnica de Galati (1990), que consistió en la siguiente secuencia: fenol puro, sosa $(\mathrm{NaOH})$ al $10 \%$ en frío, lavado con ácido acético al $10 \%$, ácido acético al $10 \%$ más dos o tres gotas de fucsina ácida, deshidratación con alcoholes graduales $(70 \%, 80 \%, 90 \%, 95 \%$ y $100 \%$ ), diafanización con creosota y montaje en bálsamo de Canadá, previa disección bajo el microscopio, estereoscopio para colocar la cabeza en posición ventral, alas extendidas, tórax lateral y abdomen lateral en machos y ventrodorsal en hembras. Se tuvo cuidado para evitar que las piezas se deformaran o perdieran la orientación. 


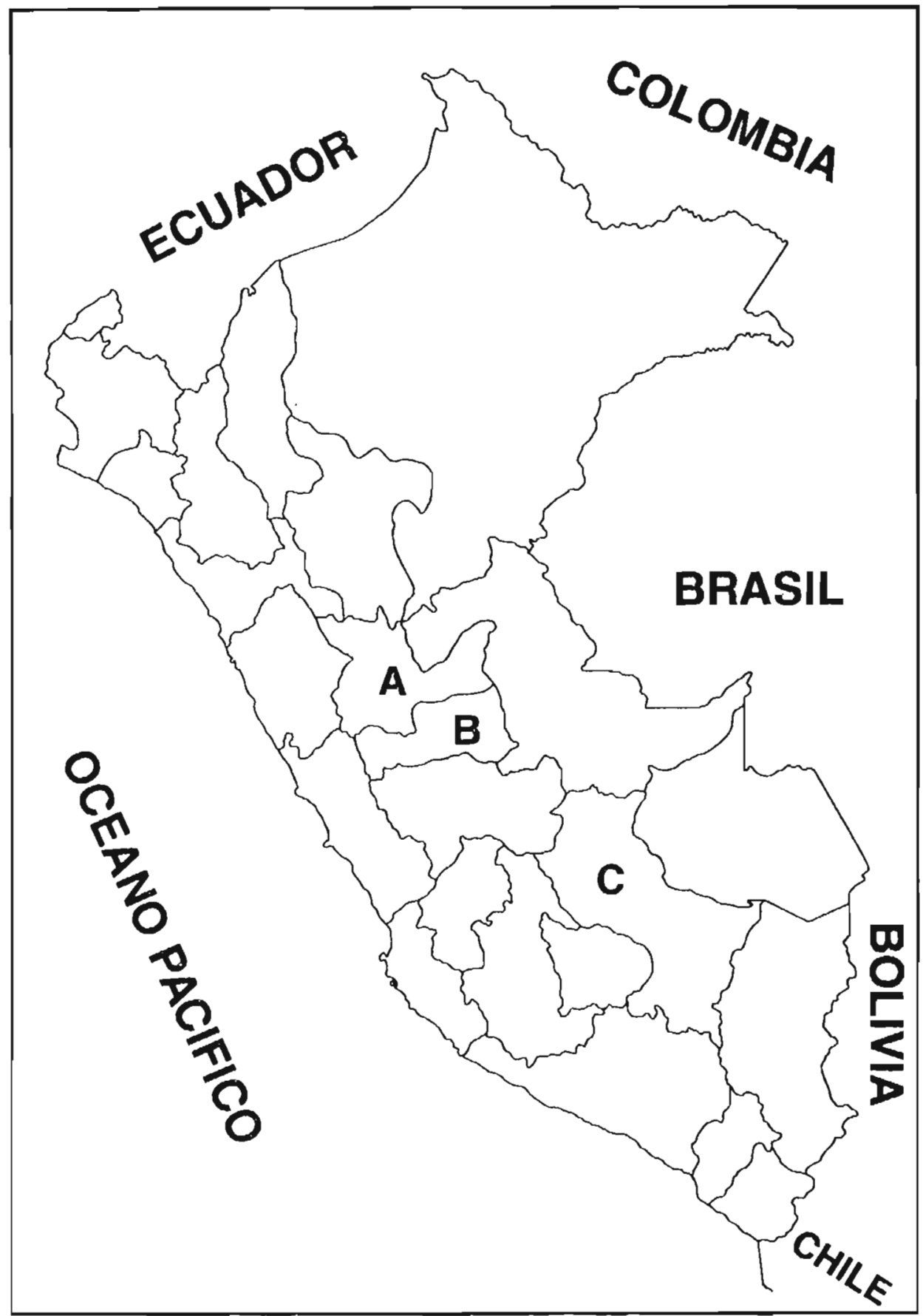

Figura 1. Ubicación de los departamentos de Huánuco (A). Pasco (B) y Cusco (C) en el Perú. 
Para identificar y organizar la lista de flebototomíneos, se ha tenido en cuenta los trabajos de Galati (1990, 1995).

\section{RESULTADOS Y COMENTARIOS}

En el presente trabajo, se incrementa 11 flebotomíneos a los 120 ya conocidos, totalizando 131 el número de flebotomíneos identificados para el Penú. Las especies que se añaden son las siguientes: Hertigiini: Warileya euniceae Fernández, Carbajal, Astete \& Wooster, 1998; W. leponti Galati \& Cáceres, 1999; Phlebotomini: Brumptomyiina: Brumptomyia quimperi Galati \& Cáceres, 1999; Sergentomyiina: Micropygomyia longipennis (Barretto, 1946); Lutzomyiina: Evandromyia sallesi(Galvão \& Coutinho, 1939), Lutzomyia rispaili Torres-Espejo, Cáceres \& Le Pont, 1995; Lutzomyia scorzai (Ortiz, 1965), Lutzomyia wattsi Fernández, Carbajal, Astete \& Wooster, 1998 y Trichophoromyia turelli Fernández, Galati,
Carbajal \& Watts, 1998; Psychodopygina: Trichophoromyia howardi (Young, 1979) y Trichophoromyia arevaloi Galati \& Cáceres, 1999.

$\mathrm{Se}$ reporta nuevos registros de flebotomíneos para los departamentos de Amazonas, Ayacucho, Huancavelica, Junín, La Libertad, Loreto, San Martín y Cajamarca (Tabla 1).

\section{Nuevos Registros de Flebotomíneos} para Perú. Lutzomyia scorzai (Ortiz, 1965) y L. rispaili Torres-Espejo, Cáceres \& Le Pont, 1995, son nuevos registros para el Perú. El primero, sólo era conocido en Venezuela y Colombia (Young and Duncan, 1994), en el Perú, está presente en la provincia de Chanchamayo (Junín). Mientras, que la segunda especie ha sido descrita para Bolivia (Torres-Espejo et al., 1995) y, en Perú está presente en localidades pertenecientes a la provincia de Calca (Cusco).

Tabla 1. Nuevo reporte para Perú y nuevos registros de flebotomineos (Diptera: sychodidae) para ocho departamentos del Perú, 2000

\begin{tabular}{|c|c|c|c|}
\hline Amazonas & La Libertad & San Martín & Ayacucho \\
\hline $\begin{array}{l}\text { E. sallesi } \\
\text { P. maranonensis }\end{array}$ & $\begin{array}{l}\text { L. caballeroi } \\
\text { W. phlebolomanica }\end{array}$ & $\begin{array}{l}\text { L. sherlocki } \\
\text { P. nevesi }\end{array}$ & $\begin{array}{l}\text { L. noguchii } \\
\text { P. aragaoi }\end{array}$ \\
\hline $\begin{array}{l}\text { P. robusta } \\
P . \text { verrucarum } \\
\text { N. yuilli yuilli } \\
\text { L. sherlocki }\end{array}$ & & P. scaffi & $\begin{array}{l}\text { T. auraensis } \\
\text { W. phlebotomanica }\end{array}$ \\
\hline Junín & Huancavelica & Loreto & Cajamarca \\
\hline $\begin{array}{l}\text { L. tejadai } \\
\text { L. scorzai } \\
\text { L. gonzaloi } \\
\text { p. nevesi } \\
\text { N. yuilli yuilli } \\
\text { N. shawi } \\
\text { P. h. hirsutus }\end{array}$ & $\begin{array}{l}\text { L. noguchii } \\
\text { W. phlebotomanica } \\
\text { P. verrucarum }\end{array}$ & $\begin{array}{l}\text { P. ayrozai } \\
P . \text { guyannensis } \\
P . \text { s. squamiventris }\end{array}$ & $\begin{array}{l}\text { P. robusta } \\
\text { P. maranonensis } \\
\text { L. ayacuchensis } \\
\text { L. castanea } \\
\text { L. pallidithorax } \\
\text { L. sallesi } \\
\text { W. phlebotomanica } \\
\text { W. lumbrerasi }\end{array}$ \\
\hline
\end{tabular}

* Nuevo reporte para Peri 
Recientemente Ogusuku et al.,1999, describe la especie L. munaypata empleando ejemplares capturados en el distrito de Santa Ana, provincia de La Convención (Cusco). Se sospecha que esta especie corresponda a $L$. rispaili, ya descrita para Bolivia (Torres-Espejo et al., 1995), pues L. munaypata y $L$. rispaili presentan características morfológicas similares, por lo que es necesario que dichos especímenes de Lutzomyia sean adaptados al laboratorio por separado y formar colonias independientes con la finalidad de obtener líneas puras y realizar cruces entre ambas; de lo contrario se debe utilizar técnicas de biología molecular ya diseñadas para taxonomía de flebotomíneos.

Departamento de Huánuco. Se registra 41 especies de flebotomíneos. De ellos ocho especies son nuevos reportes para el departamento. Además cinco especies son reportes nuevos para las provincias de Huacaybamba y Puerto Inca, cuatro para la provincia de Huamalíes, tres para Ambo, dos para las provincias de Huánuco y Pachitea, y uno para las provincias de Yarowilca y Dos de Mayo. La lista de los flebotomíneos se muestra en la

Tabla 2. Flebotomíneos presentes en el departamento de Huánuco-Perú, 2000, según Galati (1995)

\begin{tabular}{|c|c|}
\hline ESPECIES DE FLEBOTOMÍNEOS & PROVINCIAS \\
\hline \multicolumn{2}{|l|}{ HERTIGIINI Abonnec \& Leger, 1976} \\
\hline \multicolumn{2}{|l|}{ HERTIGIINA } \\
\hline \multicolumn{2}{|l|}{ WARILEYA Hertig, 1948} \\
\hline W. phlebotomanica Hertig, 1948 (3) & Huacaybamba (4) \\
\hline W. rotundipennis (Fairchild \& Hertig, 1951) (3) * & Huamalies \\
\hline \multicolumn{2}{|l|}{ PHLEBOTOMINI Rondani, 1840} \\
\hline \multirow{2}{*}{\multicolumn{2}{|c|}{$\begin{array}{l}\text { BRUMPTOMYIINA Artemiev, } 1991 \\
\text { BRUMPTOMYIA Franca \& Parrot, } 1921\end{array}$}} \\
\hline & \\
\hline B. galindoi (Fairchild \& Hertig, 1947) & Huamalíes \\
\hline B. pentacantha (Barreto, 1947) & Huamalies \\
\hline \multicolumn{2}{|l|}{ LUTZOMYIINA Abonnec \& Leger, 1976} \\
\hline \multicolumn{2}{|l|}{ SCIOPEMYIA Barreto, 1962} \\
\hline S. sordellii (Shannon \& Del Pont, 1927) & Huamalíes \\
\hline \multicolumn{2}{|l|}{$\begin{array}{l}\text { LUTZOMYIA Franca, } 1924 \\
\text { (Helcocyrtomyia) Barreto, } 1962\end{array}$} \\
\hline L. gonzaloi Ogusuku, Canales \& Pérez, 1997 & Huamalles, Puerto Inca (4) \\
\hline L. monzonensis Ogusuku, Canales \& Pérez, 1997 & Huamalíes \\
\hline L. noguchii (Shannon, 1929) (3) & Huamalies (4), Huánuco (4) \\
\hline L. pallidithorax Galati \& Cáceres, 1994 (3) & Huacaybamba (4) \\
\hline L. peruensis (Shannon, 1929) (1) & Leoncio Prado \\
\hline \multirow[t]{3}{*}{ L. tejadai Galati \& Cáceres, 1990 (1) (2) } & Ambo (4), Huacaybamba (4) \\
\hline & Huamalles (4), Pachitea (4) \\
\hline & Huánuco \\
\hline \multicolumn{2}{|l|}{ (Lutzomyia) } \\
\hline L. lichyi (Floch \& Abonnec, 1950) & Leoncio Prado \\
\hline
\end{tabular}




\section{ESPECIES DE FLEBOTOMÍNEOS}

(Tricholateralis) Galati, 1995

L. evangelistai Martins \& Fraiha, 1971

L. gomezi (Nitzulescu, 1931)

L. sherlocki Martins, Silva \& Falcão, 1971

PINTOMYIA Costa Lima, 1932

\section{(Pintomyia)}
P. fischeri (Pinto, 1926)

(Pifanomyia) Ortiz \& Scorza, 1963

$P$. nevesi (Damasceno \& Arouck, 1956)

P. serrana (Damascceno \& Arouck, 1949)

P. verrucarum (Townsend, 1913)

(2)

\section{PRESSATIA Mangabeira, 1942}

P. calcarata (Martins \& Silva, 1964)

P. choti (Floch \& Abonnec, 1941)

P. triacantha (Mangabeira, 1942)

P. trispinosa (Mangabeira, 1942)

\section{TRICHOPYGOMYIA Barreto, 1962}

T. elegans (Martins, Llanos \& Silva, 1976)

EVANDROMYIA Mangabeira, 1941

(Aldamyia) Galati, 1995

E. walkeri (Newstead, 1914) (3)

(Barrettomyia) Martins \& Silva, 1968

E. cortelezzii (Brethes, 1923)

E. sallesi (Galvão \& Coutinho, 1939)

PSYCHODOPYGINA Galati, 1995

PSATHYROMYIA Barreto, 1962

(Forattiniella) Vargas, 1978

P. aragaoi (Costa Lima, 1932)

P. barrettoi barrettoi (Mangabeira, 1942)

P. brasiliensis (Costa Lima, 1932)

$P$. coutinhoi (Mangabeira, 1942)

\section{(Xiphomyia) Artemiev, 1991}

P. ruparupa (Martins, Llanos \& Silva, 1996)

\section{PROVINCIAS}

Puerto Inca (4)

Huamalíes

Huamalies, Puerto Inca (4)

Ambo (4), Huánuco (4)

Huamalíes, Puerto Inca (4)

Huamalíes, Leoncio Prado

Huánuco, Huacaybamba (4)

Yarowilca (4), Marañón (4)

Huamalies (4)

Dos de Mayo (4)

Huamalíes

Huamalies

Huamalies

Huamalies

Huamalies

Huamalies (4)

Leoncio Prado

Ambo (4), Huánuco, Pachilea (4)
Huamalies

Huamalies

Huamalíes, Leoncio Prado

Huamalies

Huamalies 


\section{ESPECIES DE FLEBOTOMÍNEOS}

(Psathyromyia)

P. dendrophyla (Mangabeira, 1942)

P. shannoni (Dyar, 1929)

\section{VIANNAMYIA Mangabeira, 1941}

V. tuberculata (Mangabeira, 1941)

NYSSOMYIA Barreto, 1962

N. anduzei (Rozeboom, 1942)

N. antunesi (Coutinho, 1939)

N. shawi (Fraiha, Ward \& Ready, 1981) (3)

N. yuilli yuilli (Young \& Porter, 1972)

TRICHOPHOROMYIA Barreto, 1962

T. sinuosa Young \& Duncan, 1994

T. auraensis (Mangabeira, 1942)

\section{PROVINCIAS}

Huamalies, Leoncio Prado

Huamalies, Marañón

Huamalíes

Leoncio Prado

Leoncio Prado, Marañón

Puerto Inca

Huacaybamba, Leoncio Prado

\section{Flebotomíneo confundido con W. lepontiGalati \& Cáceres, 1999}

(1) Especies relacionadas como vectores de leishmaniosis

(2) Especies relacionadas como vectores potenciales de la bartonellosis humana

(3) Reportes nuevos para el departamento

(4) Nuevos reportes para la provincia

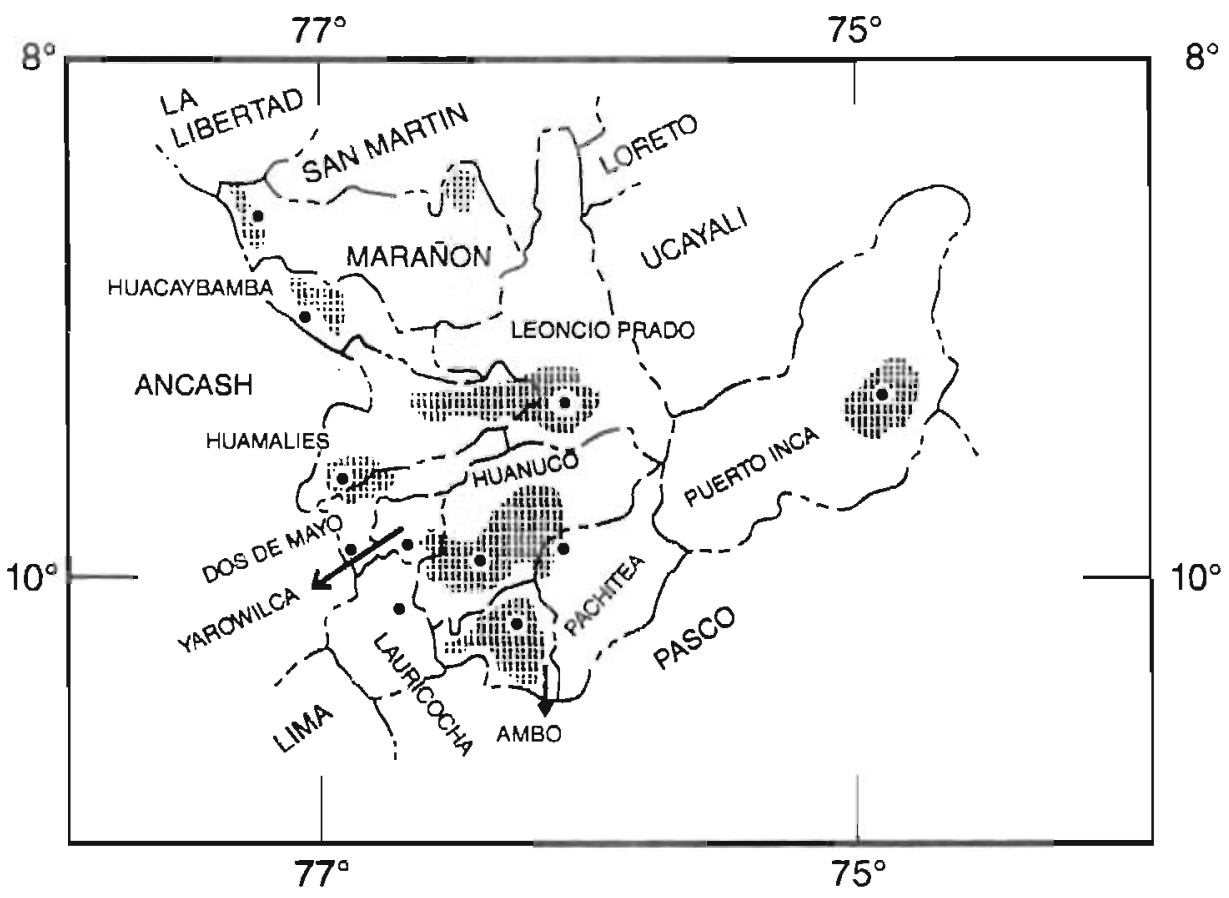

Figura 2. Once provincias del departamento de Huánuco. Se indica con puntos los lugares donde se capturó los flebotomíneos. 
Tabla 2. En la Figura 2, se indica con puntos las localidades donde se capturó los flebotomíneos en las 11 provincias de Huánuco.

Departamento de Pasco. Están identificadas 22 especies de flebotomíneos, de las cuales 12 especies son reportes nuevos para el departamento. Del mismo modo, se mencionan ocho especies de Lutzomyiina como registros nuevos en la provincia de Oxapampa y una especie para las provincias de Daniel A. Carrión y Pasco, las que se mencionan en la
Tabla 3. En la Figura 3, se observa las tres provincias del departamento de Pasco y en ellas las localidades donde se capturó los flebotomíneos.

Departamento de Cusco. Se mencionan 53 especies de flebotomíneos identificadas. De ellas, ocho especies se reportan para la provincia de La Convención. La lista de los flebotomíneos presentes en el departamento se mencionan en la Tabla 4. La Figura 4 muestra las provincias del Cusco y las zonas donde se capturó los flebotomíneos.

Tabla 3. Flebotomineos presentes en el departamento de Pasco-Perú, 2000; según Galati (1995)

\begin{tabular}{|c|c|}
\hline ESPECIES DE FLEBOTOMÍNEOS & PROVINCIAS \\
\hline \multicolumn{2}{|l|}{ HERTIGIINI Abonnec \& Leger, 1976} \\
\hline \multicolumn{2}{|l|}{ HERTIGIINA } \\
\hline \multicolumn{2}{|l|}{ WARILEYA Hertig, 1948} \\
\hline W. leponti Galati \& Cáceres, 1999 (3) & Oxapampa \\
\hline \multicolumn{2}{|l|}{ PHLEBOTOMINI Rondani, 1840} \\
\hline \multicolumn{2}{|l|}{ BRUMPTOMYIINA Artemiev, 1991} \\
\hline \multicolumn{2}{|l|}{ BRUMPTOMYIA Franca \& Parrot, 1921} \\
\hline B. quimperi Galati \& Cáceres, 1999 (3) & Oxapampa \\
\hline \multicolumn{2}{|l|}{ LUTZOMYIINA Abonnec \& Leger, 1976} \\
\hline \multicolumn{2}{|l|}{$\begin{array}{l}\text { LUTZOMYIA Franca, } 1924 \\
\text { (Helcocyrtomyia) Barreto, } 1962\end{array}$} \\
\hline L. tejadai Galati \& Cáceres, 1990 (1) (3) & Daniel A. Carrión (4), Pasco (4) \\
\hline L. osornoi (Ristorcelli \& Van Ty, 1941) & Oxapampa \\
\hline L. peruensis (Shannon, 1929) (1) & Oxapampa \\
\hline \multicolumn{2}{|l|}{ (Tricholateralle) Galati, 1995} \\
\hline L. sherlocki Martins, Silva \& Falcão, 1971 (3) & Oxapampa (4) \\
\hline \multicolumn{2}{|l|}{$\begin{array}{l}\text { MIGONEMYIA Galatl, } 1995 \\
\text { (M/gonemv/a) }\end{array}$} \\
\hline M. migonei (Franca, 1920) (3) & Oxapampa (4) \\
\hline $\begin{array}{l}\text { PINTOMYIA Costa Lima, } 1932 \\
\text { (PIfanomyla) Ortlz \& Scorza, } 1963\end{array}$ & \\
\hline P. nevesi (Damasceno \& Arouck, 1956) (3) & Oxapampa (4) \\
\hline P. nunestovari (Ortiz, 1954) (3) & Oxapampa (4) \\
\hline P. pia (Fairchild \& Hertig, 1961) & Oxapampa \\
\hline P. serrana (Damasceno \& Arouck, 1949) (2) (3) & Oxapampa (4) \\
\hline
\end{tabular}


ESPECIES DE FLEBOTOMÍNEOS

PRESSATIA Mangabeira, 1942

P. calcarata (Martins \& Silva, 1964)

EVANDROMYIA Mangabeira, 1941

(Barretomyia) Martins \& Silva, 1968

E. sallesi (Galvão \& Coutinho, 1939

Oxapampa

PSYCHODOPYGINA Galati, 1995

PSATHYROMYIA Barreto, 1962

(Forattiniella) Vargas, 1978

P. abunaensis (Martins, Falcão \& Silva, 1965) Oxapampa

(Xiphomyia) Artemiev, 1991

P. ruparupa (Martins, Llanos \& Silva, 1996) Oxapampa:

VIANNAMYIA Mangabeira, 1941

V. tuberculata (Mangabeira, 1941) (3) Oxapampa (4)

PSYCHODOPYGUS Mangabeira, 1941

P. carrerai carrerai (Barreto, 19469

Oxapampa

P. hirsutus hirsutus (Mangabeira, 1942)

Oxapampa

P. geniculatus (Mangabeira, 1941)

Oxapampa

TRICHOPHOROMYIA Barreto, 1962

T. auraensis (Mangabeira, 1942) (3)

Oxapampa (4)

T. arevaloi Galati \& Cáceres, 1999 (3)

Oxapampa

T. omagua Martins, Llanos \& Silva 1976

Oxapampa

(1) Especies relacionadas como probables vectores de leishmaniosis tegumentaria

(2) Especies relacionadas coma vectores de bartonellosis humana

(3) Reportes nuevos para el departamento

(4) Nuevos reportes para la provincia

Lutzomyia (Helcocyrtomyia) hartmanni (Fairchild \& Hertig, 1957). En 1990, Pérez et al. reportó $L$. harmanni como registro nuevo para el Perú, con ejemplares capturados en Pilcopata (Cuzco). Recientemente Ogusuku et al., 1999, describieron L. gonzaloi examinando ejemplares procedentes de Monzón (Huánuco) y comenta que los ejemplares de $L$. hartmanni capturados en Pilcopata corresponden a $L$. gonzaloi.
Warileya rotundipennis (Fairchild \& Hertig, 1951). Ogusuku et al., 1997, reportaron esta especie de flebotomíneo para el Perú en base a ejemplares capturados en localidades de la provincia de Huamalíes (Huánuco). Probablemente, este flebotomíneo ha sido confundido con W. leponti Galatí \& Cáceres, 1999, según sospecha de los autores del presente trabajo (E. A. B. G.). Warileya leponti ha sido descrita a partir de ejemplares proce- 


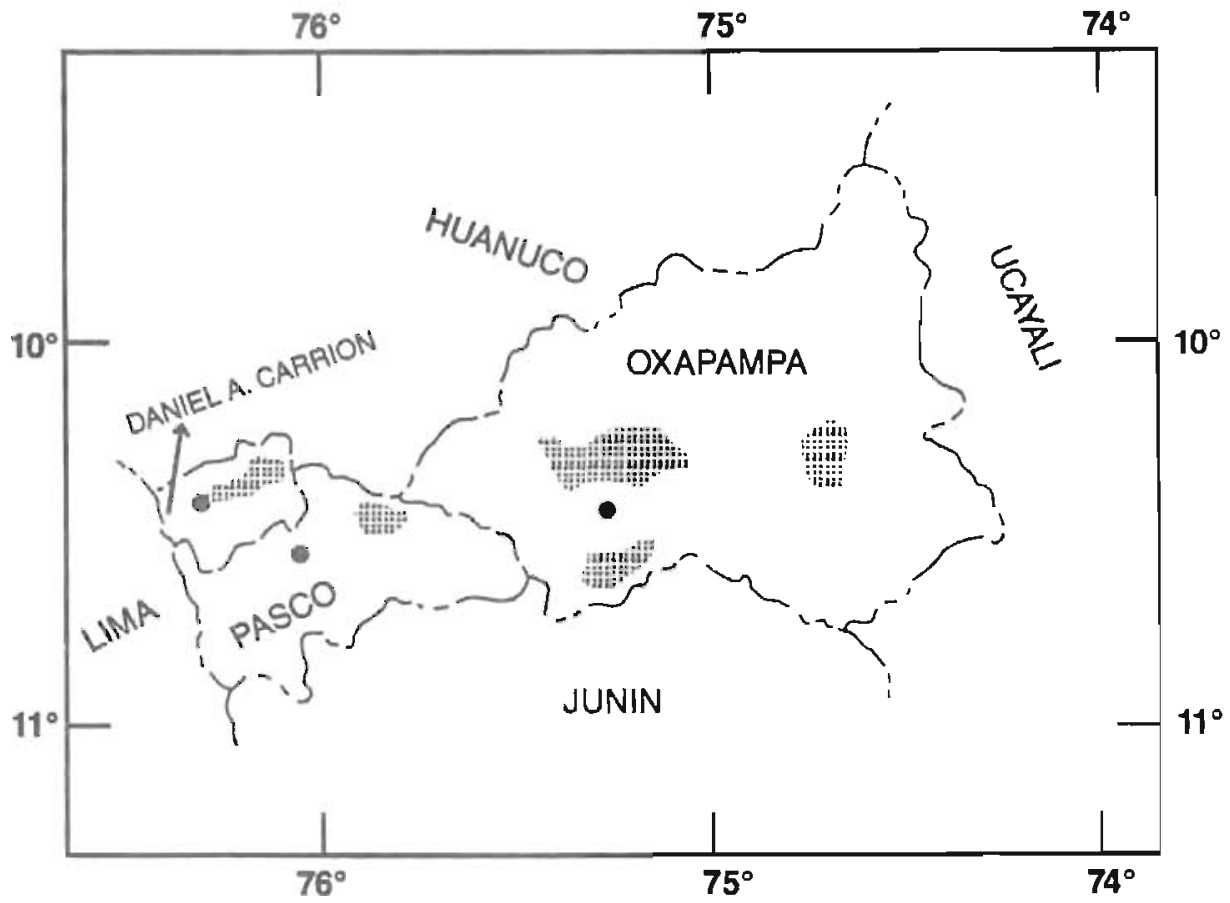

Figura 3. Tres provincias del departamento de Pasco. Las zonas punteadas señalan los lugares donde se capturó los flebotomíneos.

Tabla 4. Flebotomíneos presentes en el departamento de Cusco-Perú, 2000; según Galati (1995)

ESPECIES DE FLEBOTOMÍNEOS

SERGENTOMYIINA Artemiev, 1991

MICROPYGOMYIA Barreto, 1962

(Micropygomyia)

M. micropyga (Mangabeira, 1942)

(Sauromyia) Artemiev, 1995

M. machupicchu (Martins, Llanos \& Silva, 1975)

M. quechua (Martins, Llanos \& Silva, 1975)

M. trinidadensis (Newstead, 1922)

\section{PROVINCIAS}

Paucartambo, La Convención

Urubamba

Paucartambo, Calca, La Convención

Paucartambo, Calca,

Quispicanchis

\section{LUTZOMYIINA Abonnec \& Leger, 1976}

SCIOPEMYIA Barreto, 1962
S. preclara (Young \& Arias, 1984)
La Conveción
S. sordellii (Shannon \& Del Pont, 1927)
Paucartambo 
ESPECIES DE FLEBOTOMÍNEOS

LUTZOMYIA Franca, 1924

(Helcocyrtomyia) Barreto, 1962

L. gonzaloi Ogusuku, Canales \& Pérez, 1997

L. kirigetiensis Galati \& Cáceres, 1992 (1)

L. peruensis (Shannon, 1929) (1) (2)

L. pescei (Hertig, 1943) (1)

L. tejadai Galati \& Cáceres, 1990 (1)

L. rispaili Torres, Cáceres \& Le Pont, 1995 *

(Tricholateralis) Galati, 1995

L. gomezi (Nitzulescu, 1931)

L. sherlocki Martins, Silva \& Falcão, 1971

PINTOMYIA Costa Lima, 1932

(Pintomyia)

P. fischeri (Pinto, 1926)

(Pifanomyia) Ortiz \& Scorza, 1963

P. monticola (Costa Lima, 1932)

P. nevesi (Damasceno \& Arouck, 1956) (1)

P. nuneztovari (Ortiz, 1954) (1)

P. serrana (Damascceno \& Arouck, 1949)

$P$ verrucarum (Townsend, 1913)

PRESSATIA Mangabeira, 1942

P. calcarata (Martins \& Silva, 1964)

P. choti (Floch \& Abonnec, 1941)

P. triacantha (Mangabeira, 1942)

P. trispinosa' (Mangabeira, 1942)

EVANDROMYIA Mangabeira, 1941

(A/damyia) Galati, 1995

E. walkeri (Newstead, 1914)

(Barrettomyia) Martins \& Silva, 1968

E. cortelezzii (Brethes, 1923)

\section{PROVINCIAS}

Paucartambo

La Convención

Paucartambo, Urubamba, Calca

Quispicanchis, Cuzco,

La Convención

Calca, Urubamba, Quispicanchis

La Convención (3)

Calca.

Paucartambo, La Convención

Paucartambo, Calca,

La Convención (3)

Calca, La Convención ( 3 .

Calca

Paucartambo, Calca

La Convención

Calca, La Convención

Calca, La Convención

Paucartambo, La Convención (3)

Paucartambo

Calca

Paucartambo

Calca.

Paucartambo

Calca

Paucartambo

La Convención (3) Paucartambo 


\section{(Psathyromyia)}

P. campbelli (Damasceno, Causey \& Arouck, 1945)

Calca, La Convención

P. cuzquena (Martins, Llanos \& Silva, 1975)

P. dendrophyla (Mangabeira, 1942) Paucartambo, La Convención

P. punctigeniculata (Floch \& Abonnec, 1944) Paucartambo, La Convención Calca

Paucartambo, La Convención Calca

P. shannoni (Dyar, 1929)

Paucartambo, La Convención-

\section{PSYCHODOPYGUS Mangabeira, 1941}

P. ayrozai (Barreto \& Coutinho, 1940)

Paucartambo

P. carreraicarrerai (Barreto, 19469

Paucartambo, Calca

P. chagasi (Costa Lima, 1941)

Paucartambo

P. claustrei (Abonnec, Léger \& Fauran, 1979)

Paucartambo

P. davisi (Root, 1974)

Paucartambo, Calca,

La Convención (3)

P. geniculatus (Mangabeira, 1941)

P. hirsutus hirsutus (Mangabeira, 1942)

La Convención (3), Paucartambo

P. llanosmartinsi Fraiha \& Ward, 1980 (1)

P. nocticulus (Young, 1973)

P. panamensis (Shannon, 1926)

Paucartambo, La Convención (3)

P. yucumensis (Le Pont, Caillard, Tibayrenc \& Desjeux, 1986)

Paucartambo, La Convención (3)

Paucartambo

Paucartambo

Paucartambo

NYSSOMYIA Barreto, 1962

N. anduzei (Rozeboom, 1942)

N. shawi (Fraiha, Ward \& Ready, 1981) (3)

N. yuilli yuilli (Young \& Porter, 1972)

Paucartambo

Paucartambo

Paucartambo, La Convención (3)

TRICHOPHOROMYIA Barreto, 1962

T. auraensis (Mangabeira, 1942)

Paucartambo, Calca

T. loretonensis (Llanos, 1964)

La Convención

T. sinuosa Young \& Duncan, 1994

Paucartambo

Paucartambo, Calca

* Nuevo reporte para el Perú

(1) Especies relacionadas como vectores de leishmaniosis

(2) Especies relacionadas como vectores potenciales de la bartonellosis humana

(3) Nuevos reportes para la provincia

dentes del distrito de Villa Rica, provincia de Oxapampa (Pasco).

Especies de Flebotominos consideradas vectores potenciales de agentes patógenos de la enfermedad de Carrión y/o de la leishmaniosis tegumentaria. A continuación se menciona algunas especies de flebotomíneos consideradas vector(res) potencial(es) de agentes patógenos de la enfermedad de Carrión y leishmaniosis tegumentaria en Huánuco, Pasco y Cusco:

\section{Pintomyia (Pifanomyia) verrucarrum} (Townsend, 1913). En 1973, Tejada reportó por primera vez $P$. verrucarum para la provincia de Paucartambo (Cusco). Uno de los autores (A. G. C.), a fines de 1996, 


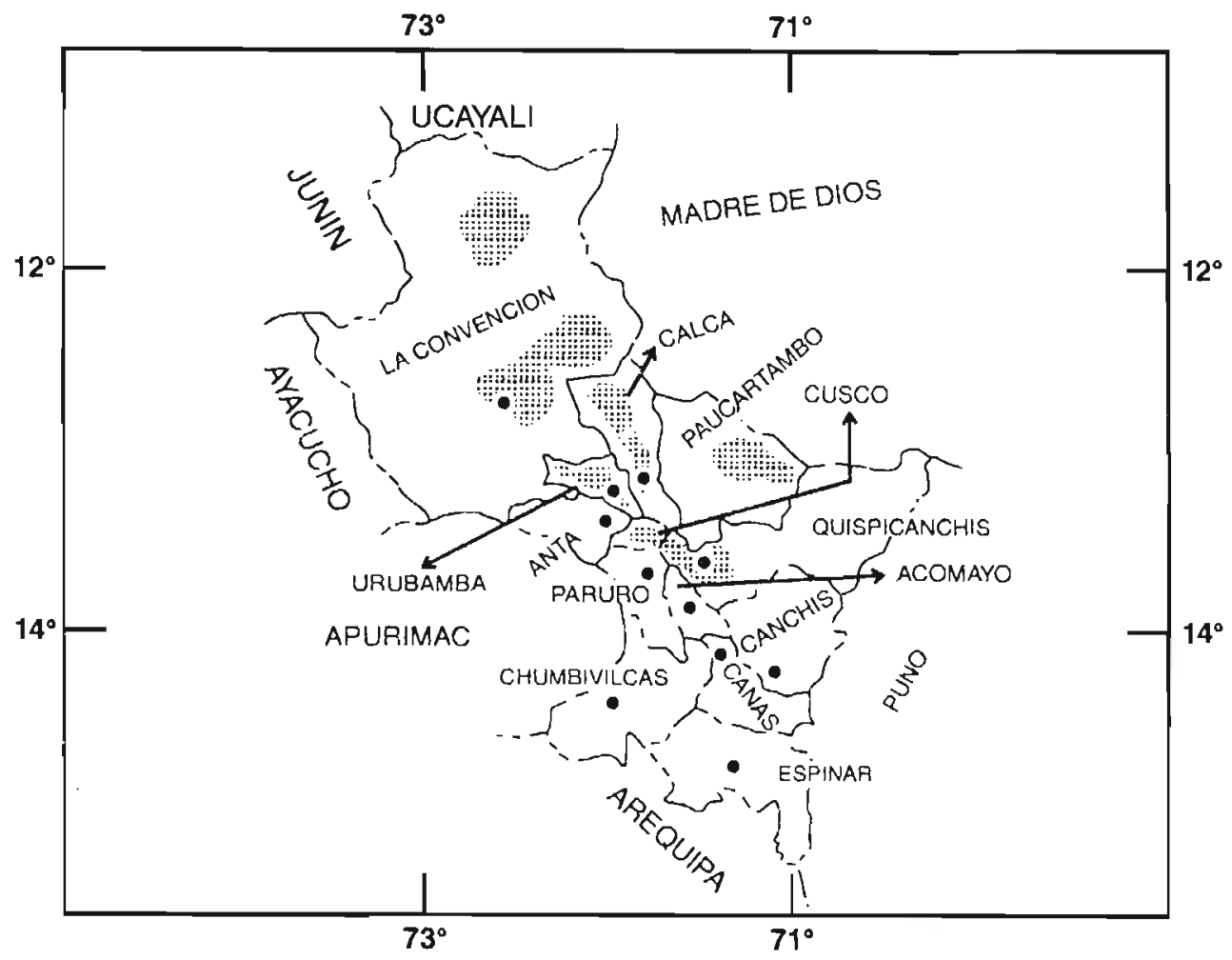

Figura 4. Trece provincias del departamento de Cusco. Con puntos se representa los lugares donde se capturó los flebotomineos.

capturó $P$. verrucarum en los alrededores de la ciudad de Quillabamba (Cusco). Es importante tener en consideración la presencia de $P$. verrucarum en esta provincia, pues esta especie es considerada vector de la enfermedad de Carrión en el Norte y Centro de los valles occidentales y ciertos valles interandinos del Norte $y$ Centro del Perú (Townsend, 1913; Hertig, 1942; Cáceres, 1993).

En localidades pertenecientes a los distritos de Pinra, Canchabamba y Huacaybamba, provincia de Huacaybamba (Huánuco) a fines de 1995 se detectaron brotes epidémicos de la enfermedad de Carrión. Simultáneamente al brote (abril-diciembre, 1996) se realizaron estudios entomológicos para determinar la diversidad y distribución geográfica de los flebotomíneos existentes en dicha zona, se capturó 175 flebotomíneos correspondien- do $96,6 \%$ a $P$. verrucarum, $1,7 \%$ a Warileya phlebotomanica, $1,1 \%$ a $L$. noguchii y $0,6 \%$ a $L$. tejadai. Se relaciona a $P$. verrucarum como el probable vector de la enfermedad de Carrión en los distritos donde se presentaron los brotes epidénicos.

Esta especie también ha sido encontrada en los alrededores de la ciudad de Huánuco, considerada por Rebagliati (1940) zona verrucógena. Así mismo, está presente en localidades del distrito de Chavinillo de la provincia de Yarowilca (Huánuco), zona potencial para que se presenten casos de enfermedad de Carrión.

Pintomyia verrucarum ha sido capturada en los alrededores del distrito de Quivilla, provincia de Dos de Mayo (Huánuco), donde se reportó cinco casos de enfermedad de Carrión entre enero y marzo de 1999. 
confirmados en el laboratorio del Hospital de Apoyo de La Unión (Oficina General de Epidemiología, 1999) yen el laboratorio de bartonellosis del Instituto de Medicina Tropical «Daniel A. Carrión» UNMSM (comunicación personal: Dr. Hugo Vizcarra).

Pintomyia verrucarum está presente en áreas rurales y urbanas de las localidades de Llata, Morca, Palanca y Nuevas Flores, jurisdicción del distrito de Llata, provincia de Huamalíes (Huánuco) localidades consideradas como zonas de riesgo para que la enfermedad de Carrión se establezca. También, se ha capturado en zonas verrucógenas del distrito de Huacrachuco, provincia de Marañón (Huánuco).

Recientemente, Andre et al., 1999, mencionaron que en ejemplares de $P$. verrucarum capturados en áreas verrucógenas del distrito de Caraz, provincia de Huaylas (Ancash), se detectó Bartonella bacilliformis mediante la técnica de Reacción en Cadena de la Polimerasa.

\section{Pintomyia (Pifanomyia) serrana} (Damasceno \& Arouck, 1949). Ha sido capturada en 16 localidades del distrito de Monzón, provincia de Huamalíes (Huánuco), donde se presentaron brotes epidémicos de enfermedad de Carrión desde 1996 hasta 1998. En estas áreas verrucógenas $P$. verrucarum está ausente, lo que nos sugiere que $P$. serrana sería el probable vector de la enfermedad de Carrion, pues en las 16 localidades donde se presentaron los brotes de la enfermedad de Carrión el $85 \%$ de flebotomíneos capturados corresponden a $P$. serrana.

Pintomyia serrana también está presente en las provincias de La Convención y Calca (Cusco), por lo que $P$. verrucarum, $P$. serrana conjuntamente con otras especies de Lutzomyiina podrían haber ocasionado los brotes de enfermedad de Carrión, que se inició en La Convención y se propagó a las provincias de Urubamba, Calca, Quispicanchis y Canchis.

\section{Lutzomyia (Helcocyrtomyia) tejadai} Galati \& Cáceres, 1990. Especie presente en cinco de 11 provincias del departamento de Huánuco. En 1991, Cáceres menciona a $L$. tejadai como vector principal de la leishmaniosis tegumentaria para la provincia de Huánuco; estudios posteriores de Ford et al., 1995, lo ratifican.

La distribución geográfica de L. tejadai está circunscrita al departamento de Huánuco. Pero también se ha reportado para algunas localidades de la provincia de La Convención (Cusco); así mismo, para Bolivia (Cáceres, 1995).

\section{Lutzomyia (Helcocyrtomyia) peruensis} (Shannon, 1929). Especie considerada vector de la leishmaniosis cutánea andina «uta» en el Norte y Centro de los valles occidentales y valles interandinos del Perú (Herrer, 1982a; Cruzado, 1987; Perez et al., 1994). Martins et al., 1976, reporta $L$. peruensis para la provincia de Leoncio Prado, sin precisar la localidad, y no está respaldado por un espécimen en ninguna colección, por lo que necesita ser confirmado este hallazgo.

Lutzomyia peruensis se halla en seis de las 13 provincias del Cusco (Fairchild and Hertig, 1957; Tejada, 1973; Cáceres, 1991); las seis provincias donde L. peruensis está presente son áreas endémicas de leishmaniosis tegumentaria, lo que implica que puede ser el vector de esta enfermedad.

EJ brote de la enfermedad de Carrión ocurrido en el departamento del Cusco se inició en el tercer trimestre de 1997 y afectó a la población ubicada a lo largo del Valle Sagrado de los Incas (Calca y Urubamba), incluyendo 
las provincias de Quispicanchis, La Convención y Canchis. En el Cusco, esta enfermedad ha ocasionado brules epidémicos en períodos comprendidos entre 1945 y 1949 y de 1950 a 1954 (Oficina General de Epidemiología, 1998). A partir de 1960 aparentemente pasó inadvertida hasta fines de 1997, pues el diagnóstico clínico de esta enfermedad, al parecer, fue confundido con hepatitis, tifoidea ofiebre amarilla silvestre.

Del 18 al 26 de abril de 1998, tres de los autores del presente trabajo (L. CH., H. Y. y A. G. C.) capturaron Lutzomyiina en 99 viviendas pertenecientes a 18 localidades de la provincia de Urubamba (Cusco). En estas y otras localidades del Valle Sagrado de los Incas los habitantes habían estado padeciendo la enfermedad de Carrión en el momento en que se realizó las capturas entomológicas. Las localidades muestreadas se ubican entre 2850 y $3100 \mathrm{msnm}$ y se mencionan a continuación: Valle Sagrado de Ccotohuincho, Rumichaca, Pampa Cocha, Chicón, San Isidro, Yanahuara, Ahumarca, Santa Inés, Miccay, Ollantaytambo, Muris, Chacchapata, Bandolista, Kowicalle, Miscana Pampa, San Isidro, Pampa Wasi y Mascabamba. En estas localidades se capturó 321 ejemplares de Lutzomyiina, de ellas $259(80,69 \%)$ se identificaron como $L$. peruensis; no fue posible identificar a $\operatorname{los} 62$ ejemplares restantes, pues se encontraban deteriorados por acción de la captura.

De lo comentado anteriormente, se sugiere que $L$. peruensis podría sei considerada probable vector de la enfermedad de Carrión en la provincia de Urubamba (Cusco), pues fue la única especie capturada en intra, peri y extradomicilios, donde los dueños habian padecido y han estado padeciendo y algunos de ellos fallecieron a causa deỉ agente etiológico de la enfermedad de Carrión.

\section{AGRADECIMIENTOS}

A las autoridades del Consejo Nacional de Ciencia y Tecnología por auspiciar proyectos de investigación relacionados con vectores de la enfermedad de Carrión y leishmaniosis tegumentaria. A los responsables de los establecimientos de Salud y Municipios que facilitaron las capturas entomológicas en La Libertad, Junín, Amazonas, Ayacucho, Loreto. Huancavelica, Pasco, Huánuco, San Martín, Cajamarca y Cusco. Al Dr. François Le Pont (O. R. S. T. O. M., Francia) por confirmar algunos especímenes. Al Sr. Roberto Morales del Centro de Salud de Llata (Huamalíes, Huánuco), al Blgo. Fernando Chapilliquen (Oficina General de Epidemiología, Ministerio de Salud) por proporcionar flebotomíneos para la identificación. AI Dr. César Náquira Velarde por la revisión del manuscrito.

\section{LITERATURA CITADA}

Andre, R.; Gordon, S.; Masuoka, P.; Korves, C.; Fernández, R.; Rejmankova, E.; Roberts, D.; Carbajal, F.; Laughlin, L.; Zyzack, M.; Gonzalis, $\mathrm{H}$ and Watts, D. 1999. Preliminary determination of the vector of human bartonellosis in Caraz, Peru through use of Elisa, PCR and remote sensing technologies. In: Ist International Conference on Bartonella as emerging pathogens. Germany, March 5-7, 1999; 33(12).

Artemiev, M. M. 1991. A classification of the subfamily Phlebotominae. Parasitologia 33 (Supp. I.): 66-77.

Cáceres, G. A. 1991. Fauna flebotómica (Diptera, Psychodidae) del valle interandino Higueras, Huánuco, Perú. Rev. per. Ent. 34: 53-54.

Cáceres, G. A. 1993. Distribución geográfica de Lutzomyia verrucarum (Townsend, 1913) (Diptera, Psychididae, Phlebotominae), vector de la bartonellosis humana en el Peru. Rev. Inst. Med. Trop. Sĩo Paulo 35: 485 490. 
Cáceres,G. A. 1995. Especies de Lutzomyia (Diptera: Psychodidae, Phlebotominae) vectores de la «uta» en el Perú. Rev. per. Ent. 38: 23-26.

Cáceres, G. A.; Galati, B. A. E; Le Pont, F: and Velasquez, C. 1997. Possible role of Lutzomyia maranonensis and Lutzomyia robusta (Diptera; Psychodidade) as vectors of human bartonellosis in three provinces of Region Nor Oriental del Marañón-Peru. 39:51-52.

Cruzado, R. L. 1987. Lutzomyia peruensis vector de Leishmania sp. en Pagash y Padai, Otuzco, Perú. In: X Congreso Latinoamericano de Microbiología. Trujillo, Perú, 14-19 Junio, 1987; 187(07).

Fairchild, G. B. and Hertig, M. 1957. Notes on the Phlebotomus of Panama. XII. The Vexator group, with a description of new species from Panama and California. Ann. Ent. Soc. Amer. 50: 325-334.

Ford, E.; Campos, P; Llanos, A. and Canales, J. 1995. Lutzomyia tejadai the suspected vector of cutaneous leishmaniasis in the unforested highlands of Huanuco department, Peru. In Second International Symposium on Phlebotomine Sandflies. September, 1995, Mérida/Venezuela. 1995: 39.

Galati, B. A. E. 1990. Sistemática dos Phlebotominae (Diptera, Psychodinae) das Américas. Tese de Doutoramen10, Faculdade de Saude Pública, Universidade de Saude de São Paulo, São Paulo., L1+275pp.

Galati, B. A. E. 1995. Phylogenetic systematics of Phlebotominae (Diptera, Psychodidade) with emphasis on American Groups. Bol. Dir. Malariol. Y San. Amb. 35 (Supp. 1):133-142.
Galati, B. A. E. \& Cáceres, A. 1999. Descrição do tres especies novas do Lutzomyia (Diptera: Psychodidae) do departamento de Pasco, Perú. Rev. Bras. Ent. 43(3/4): 293-299.

Herrer, A. 1982a. lutzomyia peruensis (Shannon, 1929), posible vector natural de la uta (leishmaniosis tegumentaria). Rev. Inst. Med. trop. São Paulo. 24: 168-172.

Hertig, M. 1942. Phlebotomus and Carrion's disease. Amer. J. Trop. Med. 22(Suppl.): $1-81$.

Llanos, Z. B. 1971. Estado actual de las enfermedades transmitidas por Phjebotomus en Latinoamérica. Bol. Soc. Entomol. Per. 6: 75-80.

Martins, V. A.; Llanos, Z. B. e Silva, J. E. 1976. Estudos sobre os flebotomineos do Peru. III. Departamento de Huánuco: Lista das espécies coletadas e descrição de duas espécies novas, Lutzomyia elegans n. sp. e Lutzomyia ruparupa n. sp. (Diptera, Psychodidade, Phlebotominae). Rev. Brasil. Biol. 36: 487-494.

Montoya, M.; Maguiña, C.; Vigo, V.; Caparó, R.; Briceño, E.; Astorga, E.; Ventocilla, P; Pérez, E, and Guerra, H. 1998. Acute bartonellosis in Regional Hospital Cusco, Peru, Abril-Junio 1998. In: 24ih International Congress of Internal Medicine. Lima, November 3-7,1998; pp. 52(033).

Noguchi, H.; Shannon, C. R; Tilden, E. B. and Tyler, J. R. 1929. Etiology of Oroya fever, XIV. The insect vectors of Carrion's disease. I. Exp. Med. 49: 993-1008.

Núñez, G.; Canales, J; Cjuno, R.; Grajeda, P. and González, C. 1998. The bartonellosis 
epidemic in the province of La Convencion, Cusco, Peru 1998. In: 24th International Congress of Internal Medicine. Lima, November 3-7,1998; pp.137(091).

Oficina General de Epidemiología, Ministerio de Salud. 1998. Epidemiología de la Bartonelosis en el Perú 1944-1998. Reporte Epidemiológico SE 32(09-15 de agosto de 1998): 14-17.

Oficina General de Epidemiología, Ministerio de Salud. 1999. Informe de Brote: Casos de bartonelosis-DISA Huánuco. Reporte Epidemiológico Semana 16(1824 de abril de 1999): 11-13.

Ogusuku, E.; Canales, J; Pérez, E. 1997. Descripción de Lutzomyia gonzaloi n. sp., y de Lutzomyia monzonensis $\mathrm{n}$. $\mathrm{sp}$. (Diptera: Psychodidae: Phlebotominae) y dos nuevos registros de Phlebotominae para el Perú. Rev. Per. Ent. 40: 71-78.

Ogusuku, E.; Chevarría, L.; Porras, R, y Pérez, E. 1999. Descripción de Lutzomyia munaypata sp. n. y Lutzomyia quillabamba sp. n. (Diptera: Psychodidae) del departamento de Cusco, Perú. Rev. Per. Ent. 41: 45-52.

Pérez, J. E.; Ogusuku, E.; Monge, J. y Young, G. D. 1990. Lutzomyia (Diptera: Psychodidae) de Pilcopata (Cusco), nuevos registros para el Perí y descripción de Lutzomyia deorsa n. sp. Rev. Per. Ent. 33: 133-135.

Pérez, J. E.; Ogusuku, E.; Inga, R.; Lopez, M.; Paz, L.; Nieto, E.; Arevalo, J. and Guerra, H. 1994. Natural Leishmania infection of Lutzomyia spp. in Peru. Trans. R. Soc. Trop. Med. Hyg. 88: 161164.
Rebagliati, R. Verruga peruana (Enfermedad de Carrión). Lima, Imprenta Torres Aguirre, 1940.

Tejada, A. 1973. Leishmaniasis tegumentaria en el Perú. Limá (Tesis de doctorado, Universidad Nacional Mayor de San Marcos).

Tesh, R. B. 1988. The genus Phlevovirus and its vectors. Ann. Rev. Ent. 33: 169-181.

Torres-Espejo, J. M., Caceres, G. A. and Le Pont, F. 1995. Description de deux nouvelles espèces de Phlebotomes du Sous-Genre Helcocyrtomyia, du piémont andin bolivien (Diptera, Psychodidae). Parasite, 2: 57-162.

Townsend, Ch. 1913a. The transmission of verruga by Phlebotomus. Amer. Med. An. 61: 1717-18.

Vizcarra, H.; Tejada, A.; Miranda, J. J. Palacios, O.; Cuadra, L. A and. Perez, J. 1999. Carrion's disease. Findings of Bartonella bacilliformis cases from the jungle of Peru. In. International Conference on Rickettsiae and Rickettsial disease, Marsella-Francia 13-16 June, 1999: 69(142).

Young, G. D y Arias, J. 1992. Flebótomos: Vectores de leishmaniasis en las Américas. Panamer. Salud, Washington DC. Cuademo Técnico N. ${ }^{\circ} 33: 1-28$.

Young, G. D. \& Duncan ,M. 1994. Guide to the identification and geographic. distribution of Lutzomyia sand flies in Mexico, the West Indies, Central and South America (Diptera: Psychodidae). Associate Publishers American. Entomological Institute. Mem. Amer. Entomol. Inst. N. ${ }^{\circ} 54,1-881$. 